\title{
Composites
}

\section{Understanding the cross-linking reactions in highly oxidized graphene/epoxy nanocomposite systems}

\author{
O. Vryonis ${ }^{1, *}$ (D) S. T. H. Virtanen ${ }^{1}$ (D), T. Andritsch ${ }^{1}$ (D), A. S. Vaughan ${ }^{1}$ (D), and P. L. Lewin ${ }^{1}$ (D) \\ ${ }^{1}$ Tony Davies High Voltage Laboratory, Department of Electronics and Computer Science, Faculty of Engineering and Physical \\ Sciences, University of Southampton, Southampton, UK
}

Received: 23 August 2018

Accepted: 25 October 2018

Published online:

31 October 2018

(C) The Author(s) 2018

\begin{abstract}
Graphene oxide (GO) was produced using acidic graphite oxidation and dispersed within an epoxy matrix using a solvent-based technique, to give nanocomposites containing up to $2 \mathrm{wt} \%$ of GO. Transmission and scanning electron microscopy revealed a fine dispersion of graphitic sheets which alters the nanocomposite's fractured surface morphology, while Fourier transform infrared spectroscopy revealed an excess of epoxide groups in the system, which are associated with the included GO. These additional moieties react with hardener amine groups and, consequently, displace the reaction stoichiometry away from the optimum. The result of this is a change in the network architecture and, in particular, the introduction of epoxy-terminated branches, which modify the dielectric $\gamma$ relaxation. During post-curing, hydroxyl groups on the GO surface react with residual epoxide groups through etherification reactions, to give a marked increase in the glass transition temperature. These reactions lead to increased interfacial interactions between the GO and the matrix, which contribute to an increase in tensile performance. In addition, post-curing also reduces the defect content within the GO lattice which, in turn, increases the electrical conductivity, dielectric permittivity and low frequency losses of the system. Associated chemical pathways are proposed.
\end{abstract}

\section{Introduction}

Graphite-based materials have long been exploited. Expanded graphite has been used as a filler [1] for polymers for over 100 years, and more recently, thin forms of graphite, termed graphite nanoplatelets (GNPs), have been utilized [2, 3]. Graphene's isolation [4] has attracted the interest of the scientific community over the last decade, due to its extraordinary mechanical, electrical and thermal properties [5]. Nevertheless, a considerable fraction of related studies [6-8] has been carried out using graphene oxide (GO), which is synthesized via graphite oxidation and exfoliation. GO is an attractive and promising filler due to its surface functionalities, which enable it to be readily dispersed in polar solvents and certain polymeric hosts [9]. The downside is that, compared with graphene, as-prepared GO

Address correspondence to E-mail: O.Vryonis@soton.ac.uk 
exhibits inferior mechanical, electrical and thermal properties, because the functional groups on its surface act as defects. Nevertheless, thermal or chemical procedures can be used to reduce the amount of impurities/functionalities and obtain performance more similar to that of graphene [10, 11].

The preparation of graphite oxide was first reported in 1859 by Brodie, through oxidation of graphite using potassium chlorate $\left(\mathrm{KClO}_{3}\right)$ and fuming nitric acid $\left(\mathrm{HNO}_{3}\right)$ [12]. This method was modified in 1898 by Staudenmaier [13] and then refined in 1958 by Hummers [14]. Some new synthesis methods, originally based on Hummers' method, have been suggested [15-18], with the two latter excluding the usage of the hazardous $\mathrm{NaNO}_{3}$. A simple, non-hazardous GO synthesis method could render it useful for cost-effective industrial applications [17]. The structure of GO is similar to that of graphene, with the difference that GO sheets are more loosely packed, with increased interlayer distance due to the presence of oxygen-containing groups. A general agreement on GO structure is that hydroxyl and epoxy groups coexist on the basal plane, while carboxylic acid groups exist on the sheet edges $[9,19]$. In spite of displaying inferior physical properties compared to pure graphene, numerous studies have used GO as the filler in epoxy-based nanocomposites, due to the improved compatibility of the constituents, which can lead to nanocomposites with high levels of dispersion and enhanced mechanical performance $[8,20,21]$.

Despite the fact that GO enhances the $T_{g}$ of epoxy nanocomposites at low filler contents [22-24], it has been reported that the functional groups on the GO surfaces may affect the epoxy cross-linking reactions at higher filler contents [25-29]. This results in modified stoichiometry and, consequently, reduced $T_{g}$ values, factors that may not be desirable. Such effects are not yet fully understood. This study therefore set out to establish a fundamental understanding of the curing reactions in epoxy systems containing GO and to evaluate consequent alterations to the system's physical attributes. For this, GO was synthesized using methods based on the process introduced by Tour et al. [18] in order to achieve high functionalization densities and was subsequently incorporated into a simple epoxy system. Characterization was conducted before and after a post-curing thermal treatment, in line with studies reported elsewhere
[30-32], in order to understand the reactions between the GO surface chemistry and the epoxy matrix.

\section{Materials and methods}

\section{Materials}

The GO nanopowder used in this study was synthesized from graphite (496596 Sigma-Aldrich), using commercially available phosphoric acid $\left(\mathrm{H}_{3} \mathrm{PO}_{4}\right.$, 79620 Sigma-Aldrich), sulphuric acid $\left(\mathrm{H}_{2} \mathrm{SO}_{4}, 435589\right.$ Sigma-Aldrich), potassium permanganate $\left(\mathrm{KMnO}_{4}\right.$, $102174 \mathrm{M}$ AnalaR) and a hydrogen peroxide solution (349887 Sigma-Aldrich). The epoxy resin was the Baxxodur $^{\circledR}$ system 5300 (BASF The Chemical Company), a two-component system using Baxxores ${ }^{\circledR}$ ER 5300 as the epoxy monomer and Baxxodur ${ }^{\circledR}$ EC 5310 as the amine-based hardener.

\section{Graphene oxide synthesis}

The oxidation process was conducted with a mixture of $\mathrm{H}_{2} \mathrm{SO}_{4}$, dried $\mathrm{H}_{3} \mathrm{PO}_{4}$ and $\mathrm{KMnO}_{4}$; the effect of including water in the mixture was also investigated. Initially, the $\mathrm{H}_{3} \mathrm{PO}_{4}$ was dried on a hot plate by heating to a boiling point of $260{ }^{\circ} \mathrm{C}$; the acid was stirred slowly throughout this process and the temperature was monitored every $10 \mathrm{~min}$. When dried, the $\mathrm{H}_{3} \mathrm{PO}_{4}$ was left at ambient conditions to cool down for several hours. The $\mathrm{H}_{3} \mathrm{PO}_{4}$ drying process was conducted in order to control the amount of $\mathrm{H}_{2} \mathrm{O}$ in the system, since the water content of commercial $\mathrm{H}_{3} \mathrm{PO}_{4}$ can vary significantly. Afterwards, $40 \mathrm{ml}$ of an $\mathrm{H}_{2} \mathrm{SO}_{4}$ and $\mathrm{H}_{3} \mathrm{PO}_{4}$ mixture was prepared in a volumetric ratio of $5: 2$ and $1.8 \mathrm{~g} \mathrm{KMnO}_{4}$ was added slowly and stirred for several minutes until a homogeneous solution was obtained. For the case of water usage in the oxidizing medium, the following process was used: the dry $\mathrm{H}_{3} \mathrm{PO}_{4}$ was slowly added to the required volume of distilled water, followed by addition of the $\mathrm{H}_{2} \mathrm{SO}_{4}$. The volumetric ratio was 5:2:2 $\left(\mathrm{H}_{2} \mathrm{SO}_{4}: \mathrm{H}_{3} \mathrm{PO}_{4}: \mathrm{H}_{2} \mathrm{O}\right)$. Afterwards, $40 \mathrm{ml}$ of the mixture and $1.8 \mathrm{~g}$ of $\mathrm{KMnO}_{4}$ were mixed, following the procedure described above.

After production of the required oxidizing medium, $300 \mathrm{mg}$ of graphite was added and the mixture was placed on a shaker and left for $4 \mathrm{~h}$ for the oxidation process to occur. After this, the mixture was neutralized, initially, by pouring it into $100 \mathrm{ml}$ of cool 
distilled water and subsequently adding $10 \mathrm{ml}$ of the $\mathrm{H}_{2} \mathrm{O}_{2}$ solution. The GO was retrieved from the solution via centrifugation (Heraeus ${ }^{\mathrm{TM}}$ Megafuge $^{\mathrm{TM}}$ 8) and, thereafter, washed with distilled water, ethanol and acetone, as follows. The GO suspension was first centrifuged at $8872 \mathrm{~g}$ for $10 \mathrm{~min}$, before the supernatant acidic solution was decanted off. The sediment was then re-dispersed in distilled water and centrifuged again using the same conditions as described above. The distilled water washing process was repeated three times in total. The same washing procedure was repeated once with ethanol and twice with acetone. A final solution of the synthesized, brown-coloured, GO in acetone was obtained and used as the filler in this study.

\section{Epoxy nanocomposite preparation}

Although it has been reported before [33] that GO/ aqueous suspensions are more stable than those based upon organic solvents, the use of an organic solvent may be beneficial for the production of nanocomposites on grounds of (a) compatibility with the polymer matrix and (b) more efficient subsequent solvent removal from the mixed system. Furthermore, non-polar organic solvents have been found to be non-compatible with GO and failed to disperse it [33], a phenomenon attributed to the high polarity of the polar oxygen groups decorating the GO surface. Therefore, acetone, which offers compatibility with both epoxy and GO (at least in the short-term [33]), was employed here as the solvent medium for exfoliation. A GO/acetone solution of $5 \mathrm{mg} / \mathrm{ml}$ for each nanofiller content was used. The epoxy monomer was added to the acetone/GO solution and probe sonicated (UP 200S) in a 100-ml glass beaker for $3 \mathrm{~h}$, using a 7-mm-diameter tip. The nanofiller contents used were $0.5,1$ and $2 \mathrm{wt} \%$ of GO, along with the unfilled reference epoxy sample (see Table 1). The next step was solvent removal using a hot plate at

Table 1 Glass transition temperatures of the unfilled and GOfilled samples before and after the post-curing

\begin{tabular}{lll}
\hline Sample & $\begin{array}{l}T_{g}\left({ }^{\circ} \mathrm{C}\right) \text { before post- } \\
\text { curing }\left( \pm 2{ }^{\circ} \mathrm{C}\right)\end{array}$ & $\begin{array}{l}T_{g}\left({ }^{\circ} \mathrm{C}\right) \text { after post- } \\
\text { curing }\left( \pm 2{ }^{\circ} \mathrm{C}\right)\end{array}$ \\
\hline Unfilled & 77.3 & 85.8 \\
$0.5 \mathrm{wt} \%$ GO & 78.2 & 85.8 \\
$1 \mathrm{wt} \%$ GO & 74.8 & 79.3 \\
$2 \mathrm{wt} \%$ GO & 66.5 & 78.3 \\
\hline
\end{tabular}

$80^{\circ} \mathrm{C}$, while slowly stirring. The procedure continued with vacuum degassing to remove any residual solvent trapped inside the system. On comparability grounds, the reference epoxy sample, despite being unfilled, was nevertheless prepared using the same solvent procedure described above, meaning that it was diluted with acetone, probe sonicated and then dried. The reason for this was to create a reference sample that took into account any effects due to traces of residual acetone; an investigation on the impact of solvent processing methods on epoxy resin is discussed elsewhere [34]. Afterwards, the hardener was added and mixed in a weight ratio of 10:2 (epoxy:hardener), in accordance with the supplier's instructions. The final mixture was then vacuum degassed to remove entrained air, cast into appropriately shaped moulds and cured in a fan oven at $70{ }^{\circ} \mathrm{C}$ for $6 \mathrm{~h}$, as suggested by the supplier. For the purposes of this study, after testing, samples were additionally post-cured in a fan oven at $130{ }^{\circ} \mathrm{C}$ for $6 \mathrm{~h}$ and tested again. In the case of destructive characterization methods (tensile testing), prior to the postcuring, new samples were prepared. It is worthy of note that, in the case of nanocomposite samples, there was a considerable colour change from brown to black after the post-curing, something that has been observed before and associated with reduction in the GO $[6,30,31]$.

\section{Characterization techniques}

Thermogravimetric analysis (TGA-PerkinElmer Pyris 1 TGA) was used to demonstrate successful functionalization of the synthesized GO and to evaluate the extent. For this, samples were first held at $60{ }^{\circ} \mathrm{C}$ for $10 \mathrm{~min}$, to remove any residual acetone, and thereafter, were heated up to $900{ }^{\circ} \mathrm{C}$ at $10{ }^{\circ} \mathrm{C} / \mathrm{min}$. All measurements were performed in a nitrogen environment. Raman spectra of the samples were obtained using a Renishaw RM1000 confocal microprobe system, operating at $6.25 \mathrm{~mW}$ power with $780 \mathrm{~nm}$ laser excitation; the tested samples were in the form of dry powder. Morphological characterization of nanocomposite samples was conducted by transmission electron microscopy (TEM-Hitachi $\mathrm{H}$ 7000), following ultramicrotomy (section thickness: $\sim 80 \mathrm{~nm}$ ) using an RMC MT-7 system and a Diatome $35^{\circ}$ diamond knife. An iD7 attenuated total reflectance Fourier transform infrared spectrometer (ATRFTIR-Nicolet iS5, Thermo-Scientific) was used to 
obtain the FTIR spectra of the samples. The respective data were normalized using a standard normal variate correction before plotting. Differential scanning calorimetry (DSC-PerkinElmer DSC7) was used in order to monitor the effect of the filler on the $T_{g}$ of the samples; these measurements involved heating, cooling and re-heating scans, all at the same heating/cooling rate of $10{ }^{\circ} \mathrm{C} / \mathrm{min}$, in order to erase the thermal history of the samples. The beneficial effects of two thermal scans are discussed elsewhere [35]. The second heating programme up to $120^{\circ} \mathrm{C}$ was used in order to determine the $T_{g}$ from the point of inflection of the step-like transition in the heat flow curve. The final $T_{g}$ value of each sample is an average of three measurements. Tensile testing was conducted using a Tinius Olsen H25KS tensometer. For this, ASTM D638-02A was followed, using dumbbellshaped samples $4 \mathrm{~mm}$ in thickness at a displacement rate of $1 \mathrm{~mm} / \mathrm{min}$, until sample failure. A total of three measurements per batch was used. The fractured areas of the tensile tested samples were examined via scanning electron microscopy (SEM) (EVO LS25, Zeiss). The electrical conductivity of the samples was measured using a Keithley 6487 picoammeter. The in-house built apparatus that was used is discussed in detail elsewhere [36]. The dielectric response of the samples was measured using a Solartron 1296 dielectric interface in combination with a Schlumberger SI 1260 impedance/phase gain analyser and a Lake Shore 332 temperature controller. The sample was mounted in a Janis Research STVP-200-XG system cryostat. Samples were gold coated to a diameter of $20 \mathrm{~mm}$, while an AC voltage of $1 \mathrm{~V}$ was used with a frequency sweep from $10^{-1}$ to $10^{5} \mathrm{~Hz}$. The average of an integration time of $5 \mathrm{~s}$ was used from $1 \mathrm{MHz}$ to $1 \mathrm{~Hz}$, while an average of 5 cycles' values was used from 1 to $0.1 \mathrm{~Hz}$. Liquid nitrogen was used for cooling and the temperature range varied from -160 to $20^{\circ} \mathrm{C}$. The software used in both cases was provided by Solartron (Solartron Materials Research and Test Software, SMaRT). The equipment has an accuracy $<0.2 \%$ in the measured impedance area but was further checked with fused silica as a reference sample [37].

\section{Results}

\section{GO characterization}

\section{TGA}

Successful graphite oxidation is demonstrated by comparison with the TGA and the differential thermogravimetric (DTG) data shown in Fig. 1 with relevant literature $[18,38]$. The graphite shows a small mass loss $(\sim 5 \%)$ at $\sim 650{ }^{\circ} \mathrm{C}$, which is ascribed to impurities, whereas both GO specimens show significant variations in mass with temperature. The pronounced mass loss $(\sim 40 \%)$ between 200 and $350{ }^{\circ} \mathrm{C}$ is associated with the removal of oxygenbased groups, with consequent production of $\mathrm{CO}$, $\mathrm{CO}_{2}$ and steam. According to DTG plot, this process is slightly shifted towards higher temperature for the
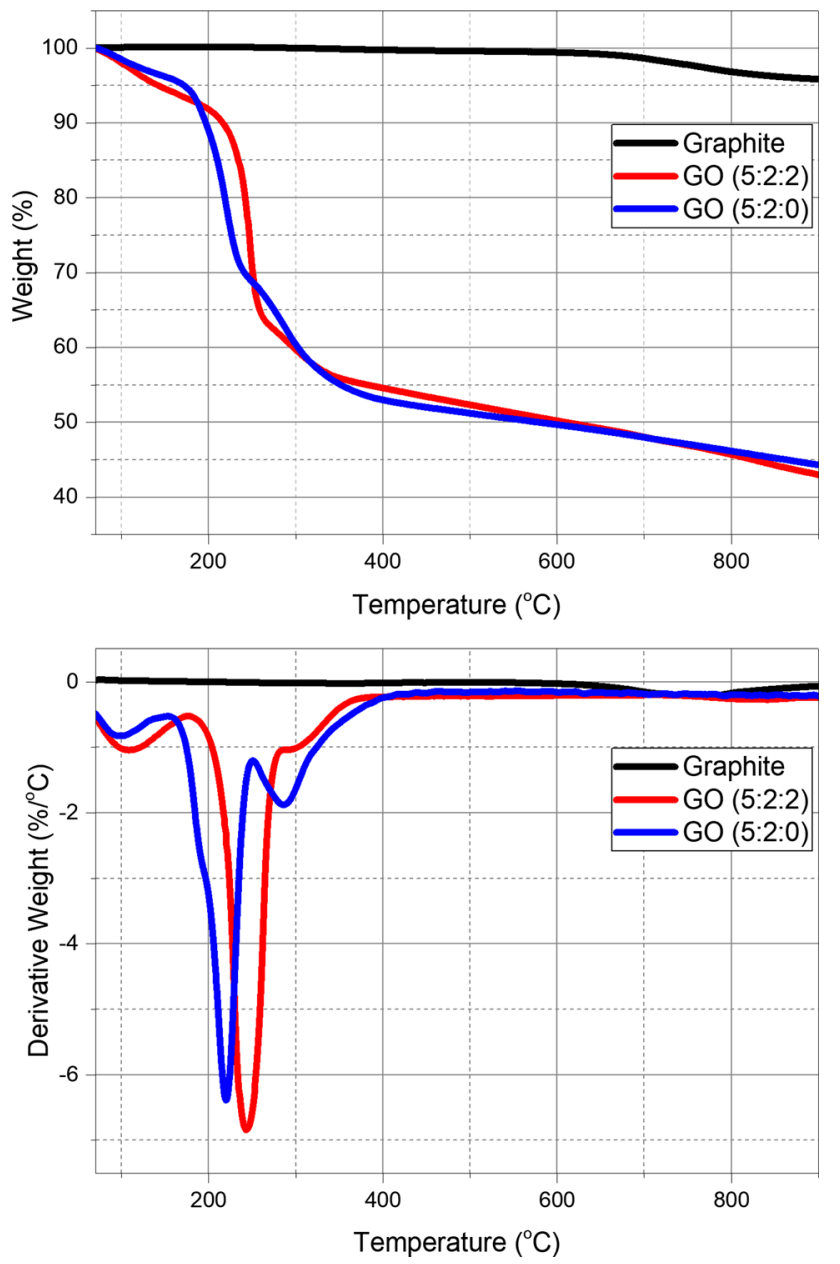

Figure 1 TGA (upper) and DTG (lower) plots for graphite (black), GO synthesized with (red) and without (blue) water in the oxidizing medium. 
GO synthesized in the presence of water. Subsequently, a further monotonic decrease in mass until the end of the measurement can be seen, which can be attributed to either the removal of more stable oxygen-based groups or combustion [18].

A smaller mass loss (5-10\%) is apparent at $\sim 100{ }^{\circ} \mathrm{C}$ for the two GO specimens, which is associated with the loss of adsorbed water [39] — water molecules can be hydrogen bonded with the hydrophilic hydroxyl and carboxyl groups [40] present on the GO surface. The water-related mass loss is slightly more pronounced for the GO synthesized with water present (indicated 5:2:2) during the oxidation process.

\section{Raman spectroscopy}

Figure 2 shows Raman spectra obtained from graphite and the two synthesized GO powders. The graphite is characterized by two bands, namely the $\mathrm{D}$ and $G$, at $\sim 1322$ and $1579 \mathrm{~cm}^{-1}$, respectively, with the $G$ band being sharper and more intense as a consequence of the dominant in-phase vibration of the graphite lattice [39]. Conversely, the GO specimen exhibits the opposite form of behaviour, with broadened and less intense $\mathrm{G}$ bands and pronounced $\mathrm{D}$ bands. As the oxidation process propagates, the graphite lattice is disrupted by the attachment of oxygen-based groups, resulting in defects that influence the Raman response in terms of the observed D band $[15,18]$.

Furthermore, the D:G intensity ratio represents an indication of the degree of disorder resulting from the chemically introduced defects [39]. The D:G intensity ratios are almost identical in the case of the two GO specimens, being 1.3, while for the graphite the same quantity is 0.77 . These results therefore confirm that the imposed oxidation process has resulted in disruption of the graphite lattice; the inclusion of water within the oxidizing medium did not, however, lead to any detectable structural differences.

\section{Structure and chemistry of epoxy/GO nanocomposite}

\section{TEM}

TEM images of the synthesized GO, dispersed in the epoxy resin, at different contents, are shown in Fig. 3.

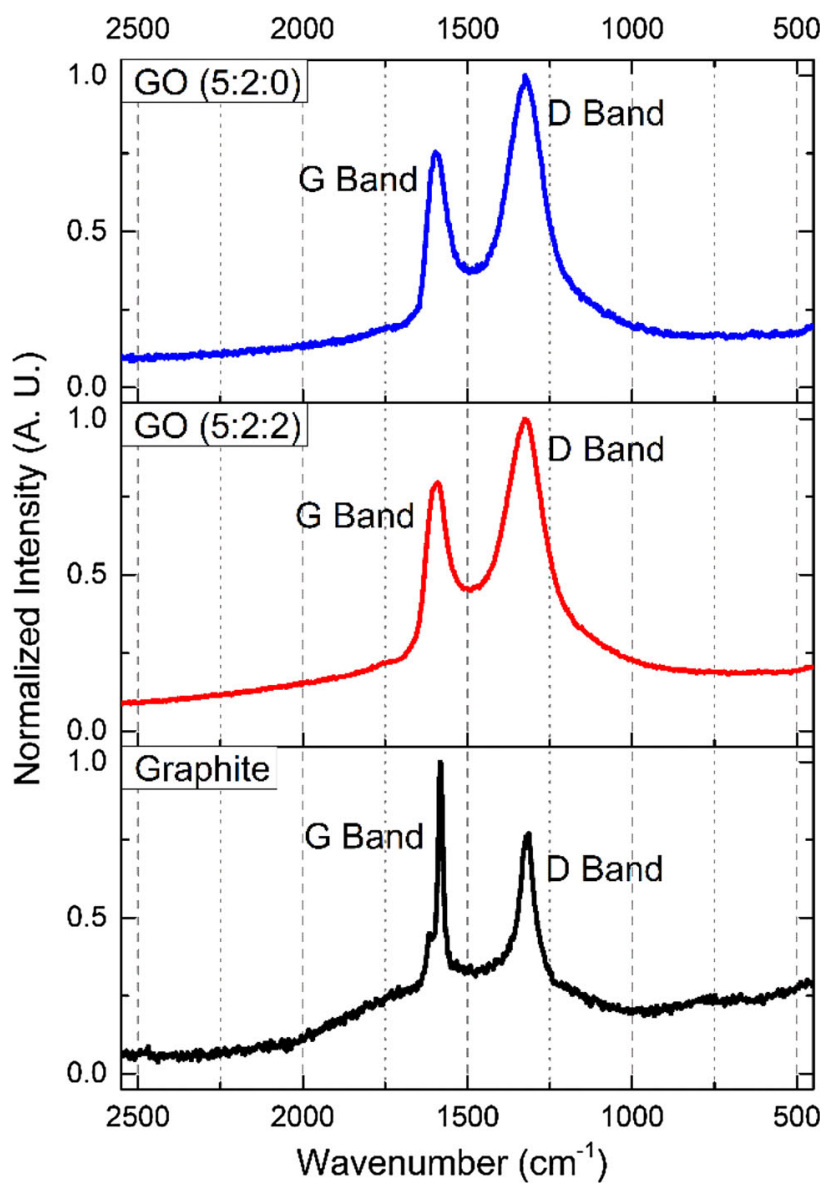

Figure 2 Raman spectra of the graphite and the GO synthesized with (red) and without (blue) water in the oxidizing medium.

Wide ellipsoidal-shaped inclusions were observed, randomly oriented, with micrometric in-plane sheet dimensions $(>2 \mu \mathrm{m})$ and nanometric sheet thicknesses $(>10 \mathrm{~nm})$. In bright field micrography of this type, contrast can arise from a number of processes, including diffraction contrast and variations in the local electron density (e.g. atomic number or thickness contrast) and we, therefore, associate the contrast variation seen here, with such effects.

\section{Epoxy cross-linking reactions}

Figure 4 presents the dominant chemical reactions that occur during amine curing of an epoxy resin. Predominantly, cross-linking occurs in such a system by epoxide ring opening through reaction with the hardener's primary (two available hydrogens) and/ or secondary (one available hydrogen) amine groups, which leads to the formation of a three-dimensional molecular network [35, 41]. The outcome of this 


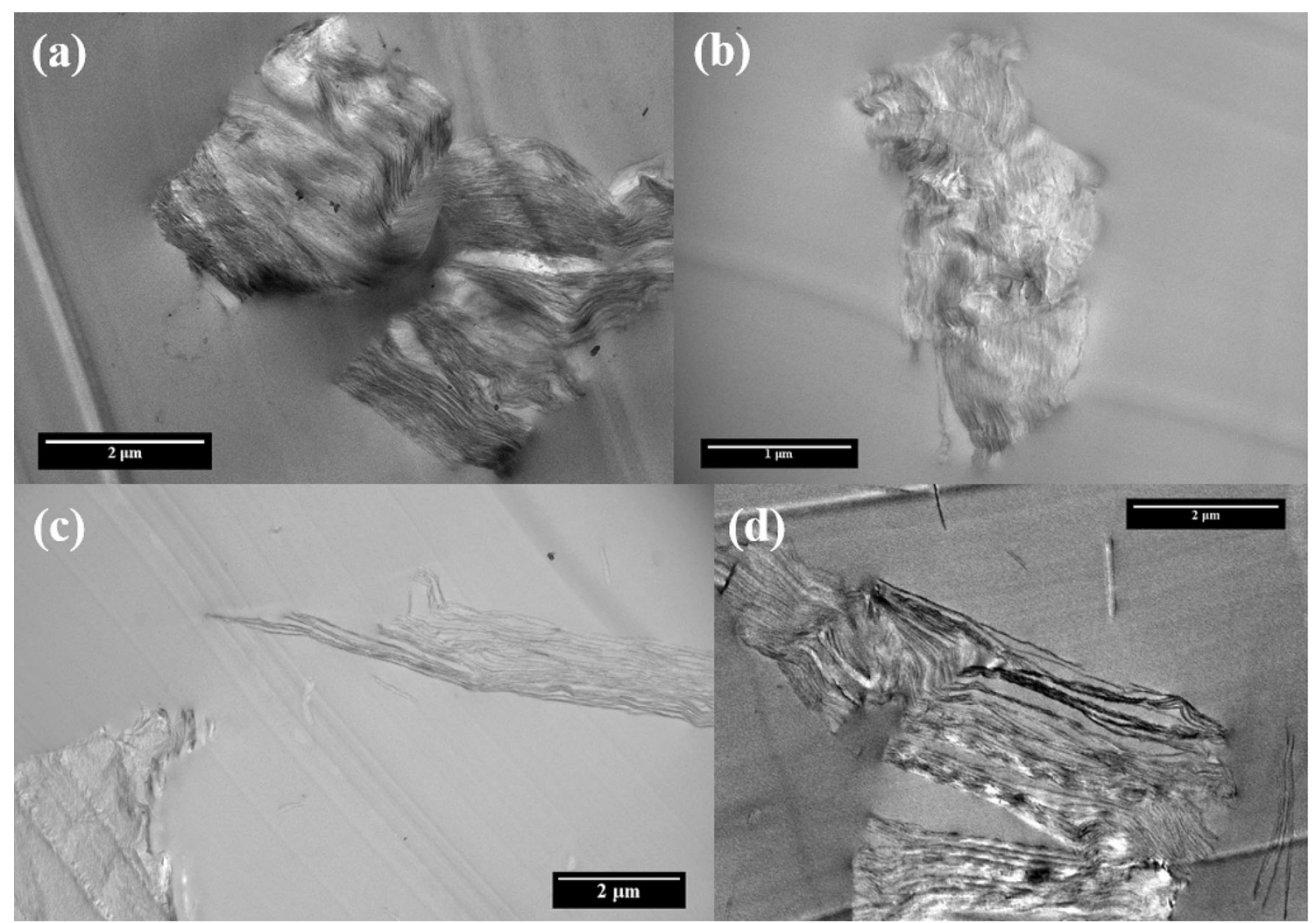

Figure 3 TEM images of the GO/epoxy nanocomposites with: $0.5 \mathrm{wt} \%$ (a), $1 \mathrm{wt} \%$ (b), and $2 \mathrm{wt} \%$ (c, d) filler contents.

reaction is the consumption of the hardener's hydrogen end groups (primary and secondary amines) and the epoxy's oxirane end groups (epoxide rings), with the consequent formation of chain structures containing pendent hydroxyl side groups (Fig. 4a, b). As the reaction proceeds, some groups remain unreacted due to the increased viscosity of the system and the consequent low molecular mobility. This phenomenon is termed vitrification [35] and is usually addressed by elevating the curing temperature (i.e. post-curing) [42]. Subsequently, at high enough temperatures (usually above $100{ }^{\circ} \mathrm{C}$ ), unreacted epoxide groups can react with the hydroxyl groups identified above and not exclusively with amine groups. In this way, more highly cross-linked macromolecular chains are formed, containing an ether linkage and a hydroxyl group (etherification or homo-polymerization of epoxy) [41], as portrayed in Fig. 4c.

\section{FTIR spectroscopy}

FTIR spectra obtained from the unfilled system and the system filled with $2 \mathrm{wt} \% \mathrm{GO}$, as well as the respective post-cured samples (unfilled and $2 \mathrm{wt} \%$ GO filled), are presented in Fig. 5. FTIR spectra of the samples containing 0.5 or $1 \mathrm{wt} \%$ of GO did not exhibit significant differences compared to that of the reference sample and, thus, are not included for the sake of brevity. The main focus here concerns three wavelength regions, $\sim 3400 \mathrm{~cm}^{-1}, 1180-1010 \mathrm{~cm}^{-1}$ and 915-830 $\mathrm{cm}^{-1}$, which are associated with hydroxyl, ether and epoxide (oxirane) groups, respectively [43-45]. The etherification reaction is confirmed by FTIR spectroscopy (Fig. 5a) in that the unfilled system displays increased absorbance in the ether group region and reduced absorbance in the oxirane region, after post-curing. The introduction of GO into the system results in pronounced oxirane group bands (Fig. 5b), which is reasonable, given that such structures are the dominant functionalities provided by the GO synthesis process used here [18]. After being post-cured, the sample filled with $2 \mathrm{wt} \%$ GO shows significantly increased absorbance in the hydroxyl group region, pronounced ether group bands and decreased oxirane bands (Fig. 5c). This indicates that the dominant reaction during postcuring is epoxide ring opening, resulting in an excess 
(a) Unreacted
oxirane<smiles>[R]CC1CO1</smiles>

(b)
Primary

amine<smiles>[R2]CN</smiles>

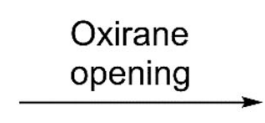

\section{Hydroxyl Secondary} group amine<smiles>[R]CCC(O)NC[R2]</smiles><smiles>[R3]CC1CO1</smiles>

(c)<smiles>[R]CC1[CH+]O1</smiles>

\section{Secondary}

amine<smiles>[R]CCC(O)NC[R]</smiles>

Secondary

(or tertiary)

amine<smiles>[R]CCC(O)NC[R]</smiles>

Hydroxyl

etherification
Figure 4 Epoxy cross-linking processes during curing (a, b) and post-curing $(\mathbf{b}, \mathbf{c})$. The reactions during curing involve epoxide ring opening via reaction with $\mathbf{a}$ primary and $\mathbf{b}$ secondary amines.

of hydroxyl groups, which is not the case for the unfilled systems, as described above. Finally, comparing the unfilled and $2 \mathrm{wt} \%$ GO-filled samples, both after being post-cured (Fig. $5 \mathrm{~d}$ ), it can be seen that the former is ether-rich and the latter hydroxylrich, while the difference in the epoxide group region is very small. This indicates that, during post-curing, residual oxiranes react both with hardener amines and with hydroxyls in the nanocomposite system.

\section{$D S C$}

The $T_{g}$ temperatures, as obtained from the respective DSC thermograms, are presented in Table 1. From this, it is evident that the incorporation of GO monotonically reduces the $T_{g}$ of the system which, in view of the FTIR data presented above, we associate with the consequent presence of an excess of epoxide groups associated with the GO. After post-curing, each sample displays an increased $T_{g}$ value, due to the propagation of cross-linking. However, the $2 \mathrm{wt} \%$ opening<smiles>[R3]CCC(O)N(C[R2])C(O)CC[R3]</smiles><smiles>[R]CCC(NC[R])OCC(O)C[R]</smiles>

During post-curing, c etherification of hydroxyls is possible, leaving unreacted amines on the reaction spot.

GO-filled sample displays a noticeably larger $T_{g}$ increase compared to the other samples, indicating the significance of the $\mathrm{GO}$ filler in the curing reactions that occur in this system. Interestingly, the sample filled with $0.5 \mathrm{wt} \%$ GO exhibits behaviour that is comparable to that of the unfilled resin, indicating that this system is still matrix dominated.

\section{Dielectric spectroscopy}

The variation of the real part of the complex relative permittivity $\left(\varepsilon^{\prime}\right)$ with frequency, obtained at $20^{\circ} \mathrm{C}$, before and after the post-curing is depicted in Fig. 6a and $b$, respectively. Since charges can accumulate around nanoparticles [46], $\varepsilon^{\prime}$ would be expected to increase with filler content. Moreover, an increased population of polar groups (existing on the GO surface) should also contribute to the dielectric response of the medium [40]. Hence, for both these reasons, a monotonic increase in $\varepsilon^{\prime}$ value with increasing GO loading level could reasonably be expected. 


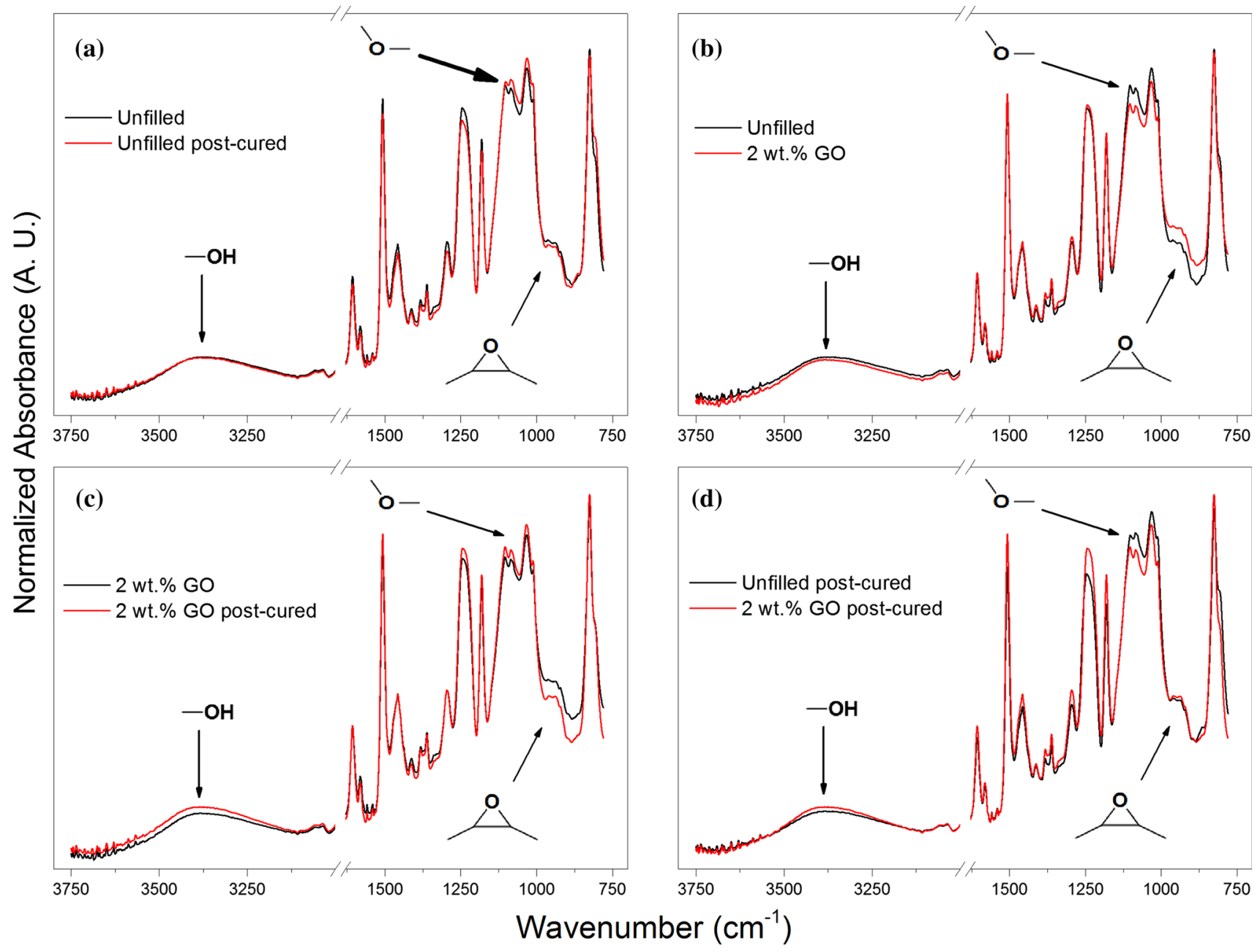

Figure 5 FTIR spectra of the unfilled and $2 \mathrm{wt} \%$ GO-filled samples, prior to and after the post-curing, in the $3750-750 \mathrm{~cm}^{-1}$ regions.

However, as shown in Fig. 6a, the sample filled with $0.5 \mathrm{wt} \% \mathrm{GO}$ is characterized by reduced $\varepsilon^{\prime}$ values compared to the unfilled sample. Nanocomposite systems exhibiting a lower permittivity than that of the unfilled matrix material have been reported and discussed elsewhere [47, 48], and this phenomenon has been ascribed to local interactions leading to molecular immobilization of interfacial regions. Thus, the low $\varepsilon^{\prime}$ values of the $0.5 \mathrm{wt} \%$ sample before postcuring could be related to such effects. After postcuring, $z^{\prime}$ is slightly increased for the unfilled sample and noticeably increased for the GO-filled samples, with the system containing $0.5 \mathrm{wt} \%$ GO now exhibiting higher values of $\varepsilon^{\prime}$ than the unfilled epoxy. It has previously been claimed that a post-curing thermal process can result in partial thermal reduction in the GO and increased values of $\varepsilon^{\prime}$ of the respective composite [30,31]. As already mentioned, reduction in GO involves removal of polar groups, which in theory should cause a decrease in permittivity. Therefore, we suggest that the dominant consequence of the post-curing is an increase in the electrical conductivity of the partially reduced GO [32] which leads to higher $\varepsilon^{\prime}$ values [48, 49], due enhanced electrical heterogeneity within the nanocomposite system [50].

Figures 7 and 8 contain imaginary relative permittivity $\left(\varepsilon^{\prime \prime}\right)$ data obtained at $-130{ }^{\circ} \mathrm{C}$ and $-70{ }^{\circ} \mathrm{C}$, respectively; these temperatures were chosen specifically so as to reveal variations in the $\gamma$ and $\beta$ relaxation processes, respectively. In the case of thermosets, the $\gamma$ and $\beta$ relaxations have been thoroughly discussed in the literature [40,50-53] and are attributed to the re-orientation of:

- Terminal groups ( $\gamma$ relaxation);

- Localized parts of the main chain ( $\beta$ relaxation). 

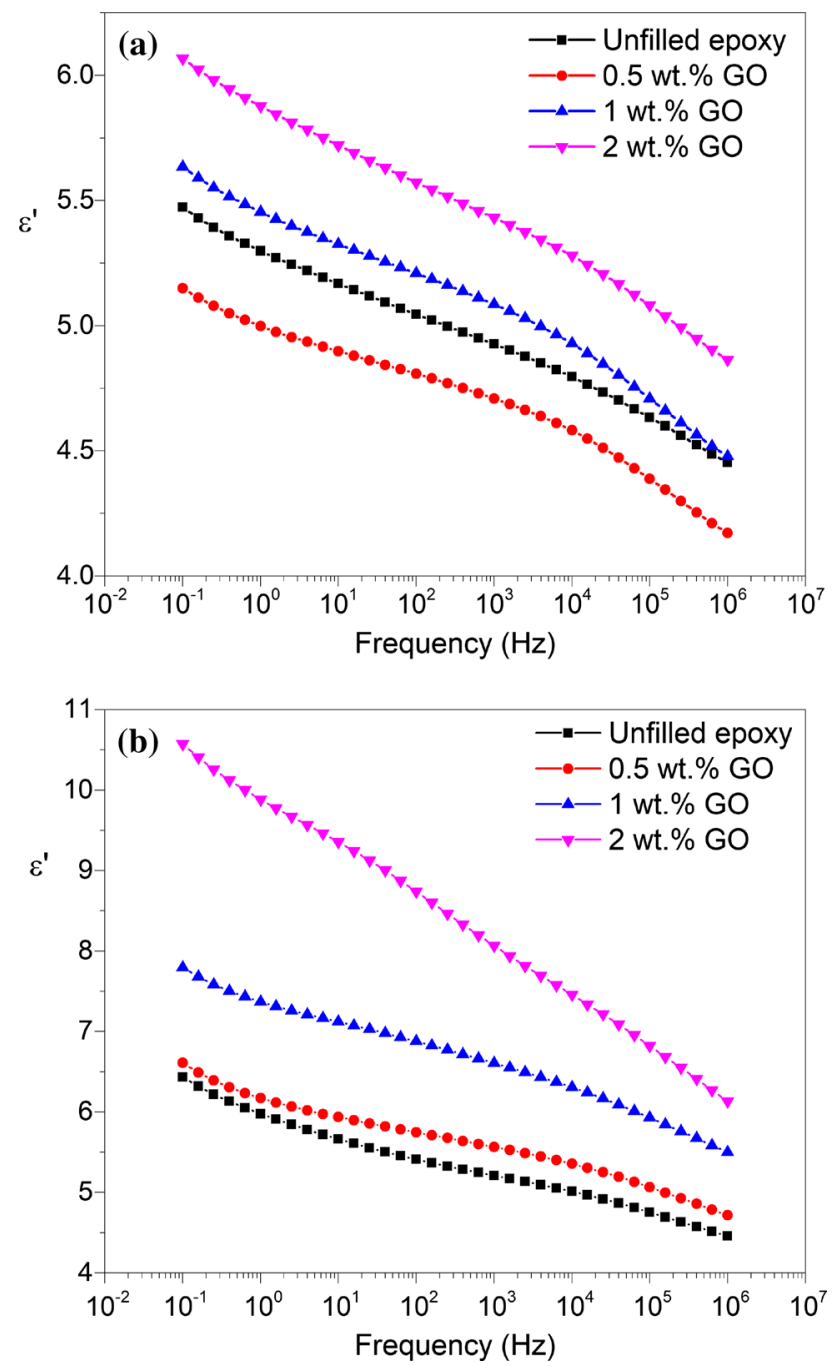

Figure 6 Real part of the relative complex permittivity at $20{ }^{\circ} \mathrm{C}$, before (a) and after (b) the post-curing.

Consider, first, the data obtained at $-130{ }^{\circ} \mathrm{C}$ (Fig. 7a). The $\gamma$ relaxation, prior to post-curing, is relatively weak for the unfilled system but broader and more pronounced for the system containing $2 \mathrm{wt} \%$ of GO. Furthermore, the $\gamma$ relaxation peak maximum of the unfilled sample is located at a lower frequency than for the system containing the GO. In general, it is considered that the $\gamma$ relaxation arises from a range of different dipolar species. In the unfilled epoxy, at the optimum stoichiometry, the observed $\gamma$ relaxation should contain contributions from equal numbers of unreacted epoxide and secondary-primary amines. In contrast, in the system containing GO, the initial excess of epoxide groups would be expected to result in the observed $\gamma$ relaxation being more strongly influenced by them. This
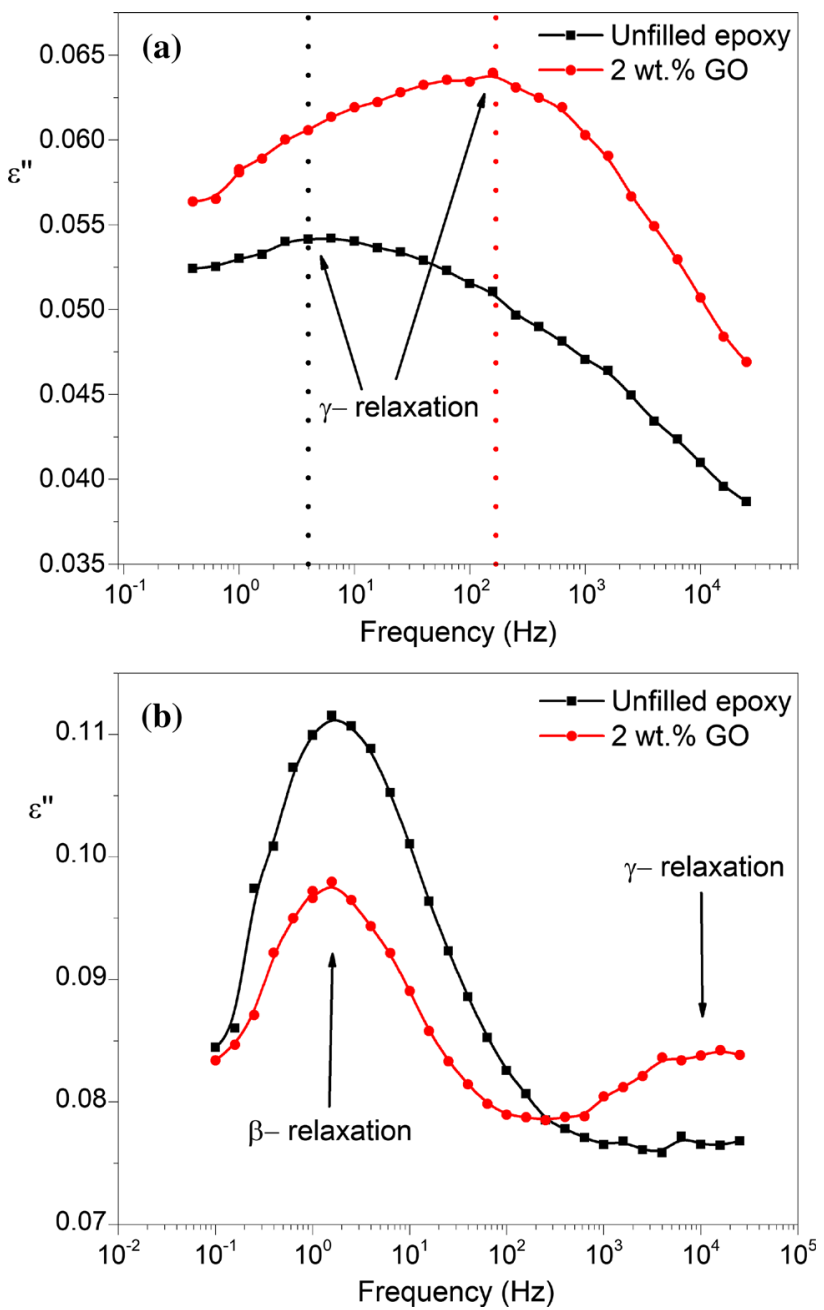

Figure 7 Imaginary part of the relative complex permittivity at $-130{ }^{\circ} \mathrm{C}(\mathbf{a})$ and $-70{ }^{\circ} \mathrm{C}(\mathbf{b})$, obtained prior to post-curing.

assertion is consistent with the reaction schemes (see Fig. 4) and FTIR data (see Fig. 5) discussed previously. As such, we suggest that for both systems, the observed $\gamma$ relaxation originates from the motion of terminal epoxide and amine groups (i.e. two different species with different dynamics and dipole moments) and that the observed differences can be explained by variations in the concentration of each.

Figure $7 \mathrm{~b}$ shows imaginary permittivity data obtained at $-70{ }^{\circ} \mathrm{C}$, which reveal the $\beta$ relaxation. This process concerns motions of hydroxyl-ether groups formed during cross-linking [40] and appears, at this temperature, as a pronounced peak located, for both systems, between 0.1 and $10 \mathrm{~Hz}$. Furthermore, an additional relaxation at $\sim 10^{4} \mathrm{~Hz}$ is evident in the system containing GO (Fig. 7b), indicated as the $\gamma$ relaxation. This attribution is based on both its 

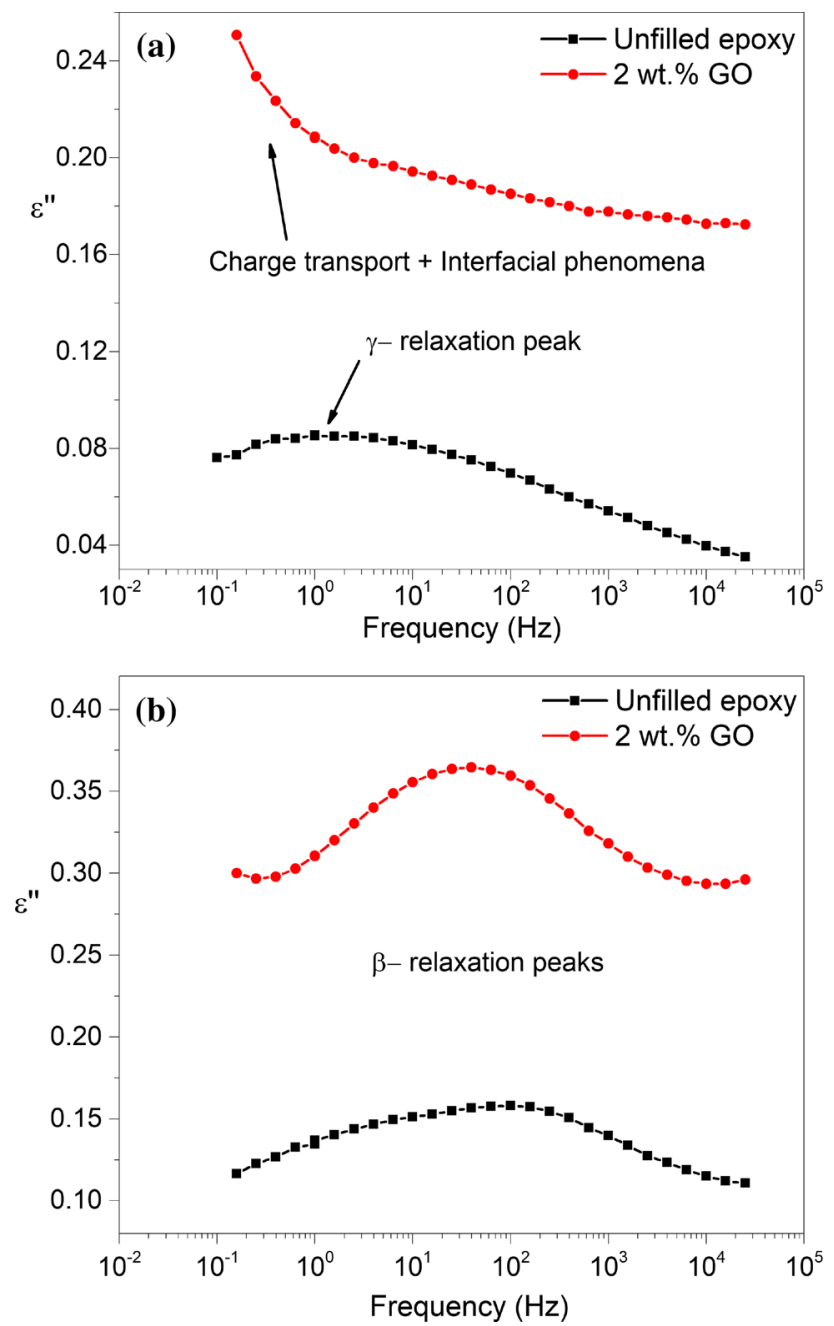

Figure 8 Imaginary part of the relative complex permittivity at $-130{ }^{\circ} \mathrm{C}$ (a) and $-30{ }^{\circ} \mathrm{C}(\mathbf{b})$ after the post-curing.

location at higher frequency than the $\beta$ relaxation and by considering dielectric spectra at intermediate temperatures (not included for the sake of brevity). To explain the presence of this relaxation only in the system containing GO, we return to our previous discussion of its molecular origins and the proposition that it stems from contributions from two different dipolar species. It is reasonable that these two dipolar motions should have different activation energies, and consequently, the $\gamma$ relaxation of the unfilled sample has shifted out of the observable frequency window (amine dominated), while the contribution stemming from epoxide groups is still observable for the GO-filled sample.

Figure 8 presents dielectric data obtained from samples after post-curing. Again, data obtained at two temperatures are shown: $-130{ }^{\circ} \mathrm{C}$ to consider the $\gamma$ relaxation and $-30{ }^{\circ} \mathrm{C}$ to consider the $\beta$ relaxation. From Fig. $8 \mathrm{a}$, the form on the $\gamma$ relaxation of the unfilled sample is comparable to that seen before post-curing (see Fig. 7a), suggesting that some unreacted secondary amines remain in the system after post-curing, where additional homo-polymerizing/ etherification reactions are possible. Conversely, post-curing of the GO-filled sample results in a dramatic change in the dielectric spectrum, with significantly increased losses being evident over the complete frequency regime, which largely swamp the $\gamma$ relaxation. We attribute this behaviour to thermal reduction in the GO leading to elimination of oxygenbased defects, enhanced GO electrical conductivity and, in turn, increased charge transport losses and/ or interfacial polarization phenomena [40, 50-53]. Post-curing also results in a modification of the $\beta$ relaxation which, from Fig. $8 \mathrm{~b}$, is now detected at higher temperatures-from $1 \mathrm{~Hz}$ to $1 \mathrm{kHz}$ at $-30{ }^{\circ} \mathrm{C}$-for both samples, which align with the published literature [40].

\section{Physical properties of epoxy/GO nanocomposite}

SEM

SEM images of the fractured tensile samples are presented in Fig. 9. From this, it is evident that the fracture surface of neat epoxy system (Fig. 9a, b) is relatively smooth, which suggests low ductility. The addition of GO results in rougher fracture surfaces (Fig. 9c-f), which is an indication of enhanced toughness caused by the presence of the GO resulting in crack propagation inhibition. These results align with previous studies and have been reported to be independent of the GO functional group density [20].

Fractured areas of the post-cured, $2 \mathrm{wt} \%$ GO-filled sample are displayed in Fig. 10. Comparing the $2 \mathrm{wt} \%$ GO-filled samples before (Fig. 9f) and after (Fig. 10a) post-curing, it can be seen that very similar surface features are displayed, implying that the same reinforcement mechanism, described above, applies here. Indeed, all the post-cured samples exhibited comparable surface textures to those seen in the respective samples prior to the thermal process, and consequently, such images are not presented here, for the sake of brevity. As expected, the presence of GO flakes was more evident in the $2 \mathrm{wt} \%$ content (Fig. 10b), and their respective sizes were 


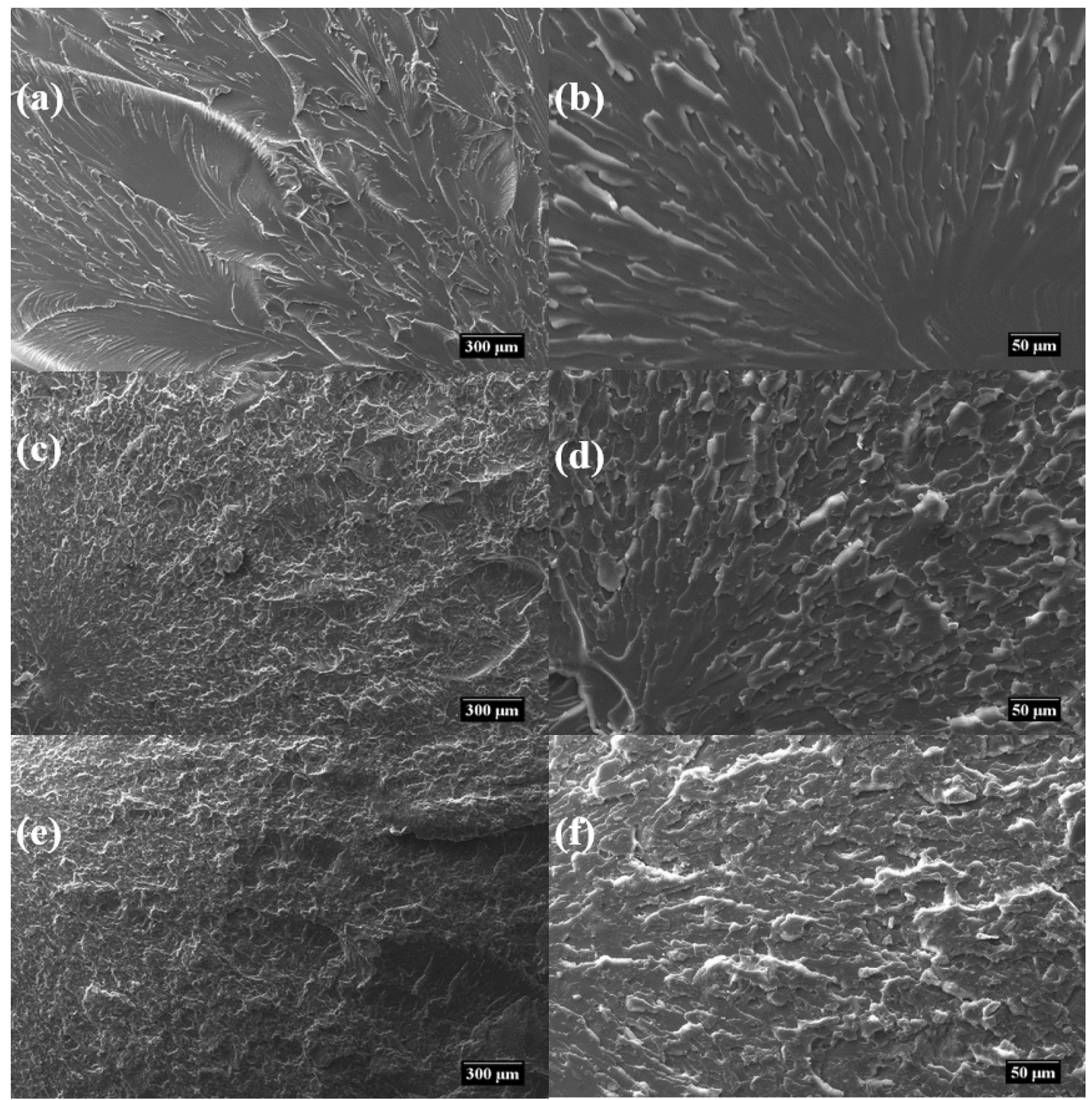

Figure 9 SEM images of the fractured areas of: $\mathbf{a}, \mathbf{b}$ unfilled epoxy; $\mathbf{c}, \mathbf{d}$ sample containing $0.5 \mathrm{wt} \%$ of GO; e, f sample containing 2 wt $\%$ GO.

found to be in good accordance with the TEM images shown previously (Fig. 3). Some degree of agglomeration or poorly distributed flakes was evident at this GO content, as shown in Fig. 10b.
Tensile testing

Figure 11 contains elastic modulus (slope of the linear part starting from $0.2 \%$ strain) and the tensile strength (maximum observed stress) data determined from tensile stress-strain curves. The addition of a small percentage of GO $(0.5 \mathrm{wt} \%)$ increases both the modulus and strength of the epoxy system, in line 

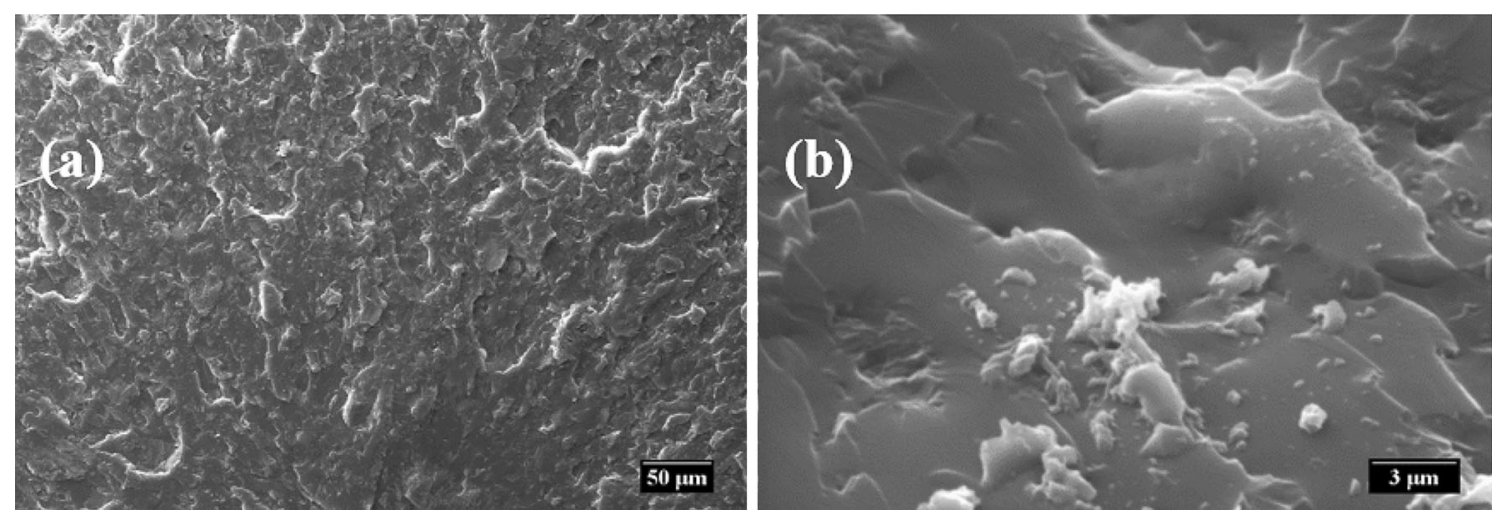

Figure 10 SEM images of the fractured areas of post-cured $2 \mathrm{wt} \%$ GO-filled samples.
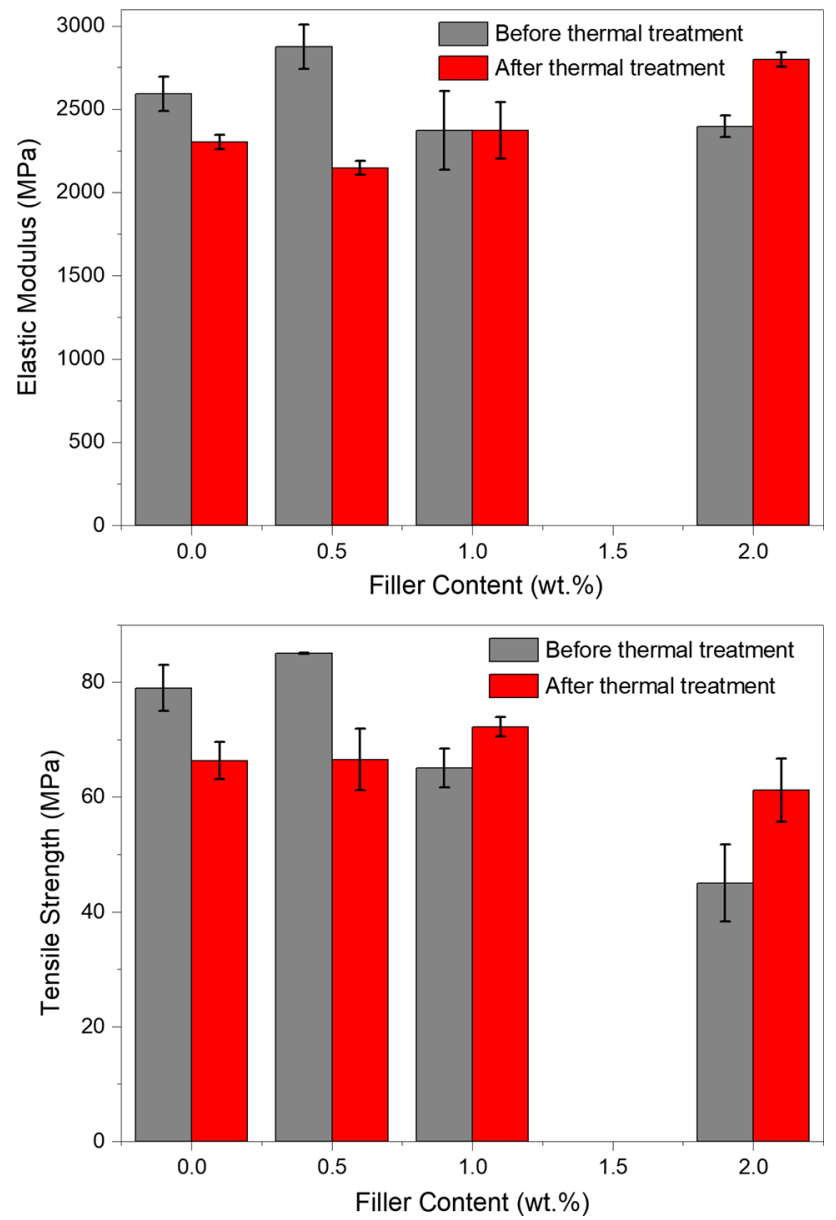

Figure 11 Elastic modulus (upper) and tensile strength (lower) of the unfilled and GO-filled samples, prior to and after post-curing. Error bars represent standard deviation.

with previous reports [20, 54, 55]. It is widely accepted that the GO interfacial adhesion with the epoxy as well as its two-dimensional structure results in enhanced modulus and reduced crack propagation, respectively [22]. This also aligns with the rough fractured surfaces seen above in the relevant SEM images. However, further addition of GO seems adversely to affect the system's performance. High GO contents have, previously, been reported to impair the storage modulus [25] and tensile strength $[21,26]$ of epoxy resin. The two latter studies consider this effect to result from the higher filler content leading to increased agglomeration, which is consistent with the SEM images shown above. Nevertheless, another factor might also be at play, which Bao et al. [25] refer to this as the "cross-linking density reduction effect", which is caused by GO hindering the epoxy/hardener interactions and leading to a deterioration in the mechanical properties of the matrix polymer. Therefore, we suggest that the disrupted stoichiometry of the matrix, caused by the increased GO content, represents another reason for this phenomenon. After post-curing, it can be seen that the tensile performance (both modulus and strength) of the unfilled system deteriorates. It has been reported previously that excessively high crosslinking densities could detrimentally affect the mechanical properties of polymers [8, 22], which might also be the case in this study after post-curing. Indeed, the suppliers do not specify any post-curing regime for the resin system used here. This phenomenon is also apparent for the $0.5 \mathrm{wt} \%$ filled system, underlying again the fact that at this GO loading level, the behaviour is matrix dominated. As the filler percentage shifts to higher values, the tensile performance starts increasing after the nanofilled samples are post-cured. In particular, the $2 \mathrm{wt} \%$ filled sample exhibits elastic modulus and tensile strength values that are noticeably higher after post-curing, a result that is counter to the behaviour of the matrixdominated samples. As mentioned earlier, apart from 
the filler dispersion effects, modifications to the matrix stoichiometry should also be considered as a deteriorating factor. Therefore, we suggest that the observed enhancement in mechanical performance seen at higher GO loading levels is stoichiometry related, since the GO dispersion within the matrix would not improve during post-curing.

\section{Electrical conductivity}

Figure 12 compares electrical conductivity data obtained from the unfilled and GO-filled samples, before and after post-curing. It can be seen that initially, prior to post-curing, the incorporation of GO does not alter the conductivity of the system while, after the post-curing, all samples exhibit increased electrical conductivity values. However, the GO-filled systems show a much more pronounced increase compared to the unfilled system, suggesting that this uplift is not determined by changes in the matrix polymer. Rather, we suggest that, for the nanofilled systems, thermal reduction in the GO is the dominant explanation for the increased electrical conductivity values that are seen [32].

\section{Discussion}

Considering the FTIR and DSC data obtained from the nanocomposite systems before post-curing, it is evident that the presence of the heavily oxidized GO

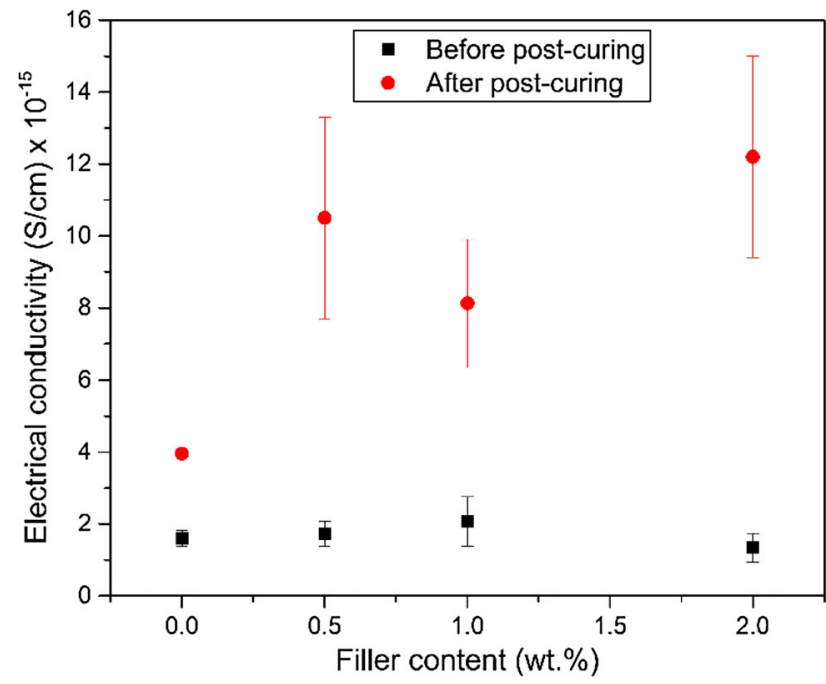

Figure 12 Electrical conductivity of the unfilled and GO-filled samples, prior to and after the post-curing. affects the epoxy cross-linking reaction by introducing additional oxirane groups, which consumes the hardener amine groups. Chemically, the included GO acts in a comparable way to the inclusion of an excess of epoxy monomer, and the net result is a network structure that contains epoxy-terminated branches. The consequence of this less constrained molecular architecture is a progressive decrease in $T_{g}$ with increasing GO content and, in the case of the $2 \mathrm{wt} \%$ filled sample, pronounced oxirane-related FTIR bands. During post-curing, while the previously mentioned reactions may still occur, additionally, hydroxyl groups become active and consume oxirane groups in the matrix via etherification. Such reactions will occur both within the matrix polymer and at GO surfaces. In this way, post-curing consumes excess epoxide groups and, in respect, can be considered to compensate for the initial stoichiometry imbalance within systems containing GO. The consequences of this are the observed increase in $T_{g}$ and the pronounced ether-related FTIR bands in the 2 wt\% GOfilled sample after being post-cured. These reactions potentially enhance the bonding between the epoxy resin and the GO, resulting in improved tensile performance values for a system that would otherwise be thermally deteriorated after the post-curing process.

Nevertheless, we suggest that the initial excess of epoxide groups cannot be fully consumed, since complete reaction would manifest itself through: (a) elevated $T_{g}$ and (b) increased ether-related FTIR bands, compared to the unfilled sample, which is not the case. However, the FTIR spectrum of the $2 \mathrm{wt} \%$ filled sample shows similar (albeit slightly stronger) oxirane-related FTIR bands with the unfilled system (Fig. 5d) despite the fact that the nanocomposite system's oxirane groups cannot be consumed to the same extent. The explanation of this is provided by the colour change of the filler, the increased real and imaginary part of permittivity and the reported electrical conductivity data, all of which indicate reduction in the GO during post-curing. The proposed reaction scheme is presented in Fig. 13.

\section{Conclusion}

Graphene oxide was successfully synthesized using acidic graphite oxidation, since TGA and Raman characterization conducted on the resulting GO both 
(a) Graphene oxide

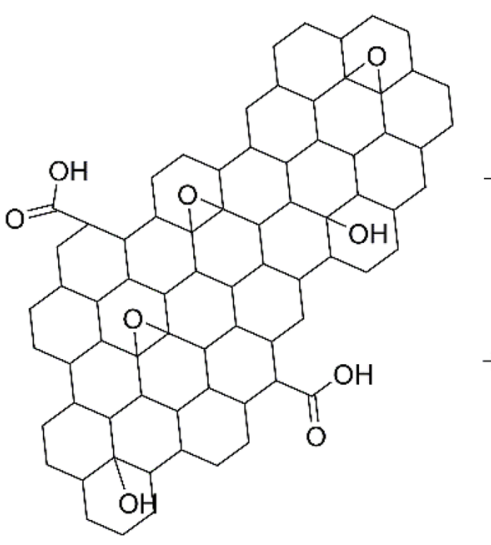

(b)

$$
\text { Graphene oxide with secondary }
$$
amine attached<smiles>[R3]CC1CO1</smiles>

Figure 13 Cross-linking reactions between epoxy and GO. During curing a GO surface epoxide groups react with hardener molecules forming a hydroxyl and a secondary amine on the

indicate attachment of a large amount of oxygencontaining groups. Additionally, the inclusion of water in the oxidizing agent has been shown not to provide any significant alteration on the consequent density of functional groups formed. Epoxy-based nanocomposites were prepared, with GO filler contents of up to $2 \mathrm{wt} \%$. Examination of these samples by TEM imaging showed a fine dispersion of micrometric-sized planar graphitic sheets, while SEM imaging demonstrated rough fractured surfaces for all GO-filled samples. FTIR spectroscopy revealed an excess of epoxide ring groups in the system, which we associate with the GO. The presence of these additional reactive moieties has important consequences, in that they consume hardener amine groups (acting as excess epoxy monomer) and,
Secondary amine attached and formation of hydroxyl group

Excess unreacted oxirane<smiles>[R1]CC1CO1</smiles>

Epoxy post-curing $\left(>100^{\circ} \mathrm{C}\right)$

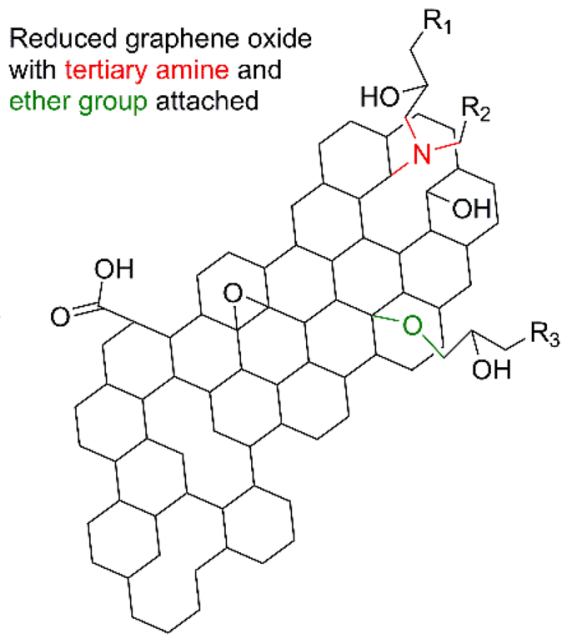

reaction site. During post-curing $\mathbf{b}$ the epoxide-terminated groups can react either with secondary amines or with hydroxyl groups through etherification. GO is also being partially reduced.

consequently, displace the overall reaction stoichiometry away from the optimum. The result of this is a change in the network architecture and, in particular, the introduction of epoxy-terminated branches; this change was observed in decreased $T_{g}$ and tensile performance and was confirmed by dielectric $\gamma$ relaxation. During post-curing, the hydroxyl groups existing on the GO surface react with residual epoxide groups through etherification reactions. Accordingly, post-curing leads to a large increase in $T_{g}$ for the $2 \mathrm{wt} \%$ GO-filled system compared with the unfilled epoxy. These reactions lead to increased interfacial interactions at GO surfaces, which contribute to the observed enhancement in tensile performance seen in the GO systems after post-curing. Furthermore, post-curing also thermally reduces the 
GO which, in turn, increases the electrical conductivity, dielectric permittivity and losses at low frequencies of the system. We therefore infer that the post-curing process serves: (a) to promote the propagation of cross-linking through two different reaction routes and (b) to reduce partially the GO. Associated chemical pathways are proposed.

\section{Acknowledgements}

This project has received funding from the European Union's Horizon 2020 research and innovation programme under the Marie Sklodowska-Curie Grant Agreement No. 642771.

\section{Compliance with ethical standards}

Conflict of interest The authors declare that they have no conflict of interest.

Open Access This article is distributed under the terms of the Creative Commons Attribution 4.0 International License (http://creativecommons.org/ licenses/by/4.0/), which permits unrestricted use, distribution, and reproduction in any medium, provided you give appropriate credit to the original author(s) and the source, provide a link to the Creative Commons license, and indicate if changes were made.

\section{References}

[1] Celzard A, Marêché JF, Furdin G (2002) Surface area of compressed expanded graphite. Carbon N Y 40:2713-2718. https://doi.org/10.1016/S0008-6223(02)00183-5

[2] Jang BZ, Zhamu A (2008) Processing of nanographene platelets (NGPs) and NGP nanocomposites: a review. J Mater Sci 43:5092-5101. https://doi.org/10.1007/s10853008-2755-2

[3] Kalaitzidou K, Fukushima H, Drzal LT (2007) A new compounding method for exfoliated graphite-polypropylene nanocomposites with enhanced flexural properties and lower percolation threshold. Compos Sci Technol 67:2045-2051. h ttps://doi.org/10.1016/j.compscitech.2006.11.014

[4] Novoselov KS, Geim AK, Morozov SV et al (2004) Electric field in atomically thin carbon films. Science 306:666-669. h ttps://doi.org/10.1126/science.1102896
[5] Stankovich S, Dikin DA, Dommett GHB et al (2006) Graphene-based composite materials. Nature 442:282-286. htt ps://doi.org/10.1038/nature04969

[6] Young RJ, Kinloch IA, Gong L, Novoselov KS (2012) The mechanics of graphene nanocomposites: a review. Compos Sci Technol 72:1459-1476. https://doi.org/10.1016/j.compsc itech.2012.05.005

[7] Potts JR, Dreyer DR, Bielawski CW, Ruoff RS (2011) Graphene-based polymer nanocomposites. Polym (Guildf) 52:5-25. https://doi.org/10.1016/j.polymer.2010.11.042

[8] Atif R, Shyha I, Inam F (2016) Mechanical, thermal, and electrical properties of graphene-epoxy nanocomposites - a review. Polym (Basel). https://doi.org/10.3390/ polym8080281

[9] Bhattacharya M (2016) Polymer nanocomposites-a comparison between carbon nanotubes, graphene, and clay as nanofillers. Mater (Basel). 9:1-35

[10] Pei S, Cheng H-M (2012) The reduction of graphene oxide. Carbon N Y 50:3210-3228. https://doi.org/10.1016/j.carbon. 2011.11.010

[11] Stankovich S, Dikin DA, Piner RD et al (2007) Synthesis of graphene-based nanosheets via chemical reduction of exfoliated graphite oxide. Carbon N Y 45:1558-1565. https://doi. org/10.1016/j.carbon.2007.02.034

[12] Brodie BC (1859) On the atomic weight of graphite. Philos Trans R Soc Lond 149:249-259. https://doi.org/10.1098/rstl. 1859.0013

[13] Staudenmaier L (1898) Verfahren zur darstellung der graphitsäure. Berichte der Dtsch Chem Gesellschaft 31:1481-1487. https://doi.org/10.1002/cber.18980310237

[14] Hummers WS, Offeman RE (1958) Preparation of graphitic oxide. J Am Chem Soc 80:1339

[15] Kosynkin DV, Higginbotham AL, Sinitskii A et al (2009) Longitudinal unzipping of carbon nanotubes to form graphene nanoribbons. Nature 458:872-876. https://doi.org/10. 1038/nature 07872

[16] Higginbotham AL, Kosynkin DV, Sinitskii A et al (2010) Lower-defect graphene oxide nanoribbons from multiwalled carbon nanotubes. ACS Nano 4:2059-2069. https://doi.org/ $10.1021 / \mathrm{nn} 100118 \mathrm{~m}$

[17] Chen J, Yao B, Li C, Shi G (2013) An improved hummers method for eco-friendly synthesis of graphene oxide. Carbon N Y 64:225-229. https://doi.org/10.1016/j.carbon.2013.07. 055

[18] Marcano DC, Kosynkin DV, Berlin JM et al (2010) Improved synthesis of graphene oxide. ACS Nano 4:4806-4814. https://doi.org/10.1021/nn1006368

[19] Krishnamoorthy K, Veerapandian M, Yun K, Kim SJ (2013) The chemical and structural analysis of graphene oxide with 
different degrees of oxidation. Carbon N Y 53:38-49. http s://doi.org/10.1016/j.carbon.2012.10.013

[20] Li Z, Young RJ, Wang R et al (2013) The role of functional groups on graphene oxide in epoxy nanocomposites. Polym (Guildf) 54:5821-5829. https://doi.org/10.1016/j.polymer.2 013.08.026

[21] Wan Y, Tang L, Gong L et al (2013) Grafting of epoxy chains onto graphene oxide for epoxy composites with improved mechanical and thermal properties. Carbon N Y 69:467-480. https://doi.org/10.1016/j.carbon.2013.12.050

[22] Wang X, Jin J, Song M (2013) An investigation of the mechanism of graphene toughening epoxy. Carbon N Y 65:324-333. https://doi.org/10.1016/j.carbon.2013.08.032

[23] Park YT, Qian Y, Chan C et al (2015) Epoxy toughening with low graphene loading. Adv Funct Mater 25:575-585. h ttps://doi.org/10.1002/adfm.201402553

[24] Silva LCO, Silva GG, Ajayan PM, Soares BG (2015) Longterm behavior of epoxy/graphene-based composites determined by dynamic mechanical analysis. J Mater Sci 50:6407-6419. https://doi.org/10.1007/s10853-015-9193-8

[25] Bao C, Guo Y, Song L et al (2011) In situ preparation of functionalized graphene oxide/epoxy nanocomposites with effective reinforcements. J Mater Chem 21:13290-13298. h ttps://doi.org/10.1039/c1jm11434d

[26] Galpaya D, Wang M, Yan C et al (2013) Fabrication and characterisation of graphene oxide-epoxy nanocomposite. In: Fourth international conference on smart materials and nanotechnology in engineering, p 8793

[27] Tang X, Zhou Y, Peng M (2016) Green preparation of epoxy/graphene oxide nanocomposites using a glycidylamine epoxy resin as the surface modifier and phase transfer agent of graphene oxide. ACS Appl Mater Interfaces 8:1854-1866. https://doi.org/10.1021/acsami.5b09830

[28] Zhou T, Nagao S, Sugahara T et al (2015) Facile identification of the critical content of multi-layer graphene oxide for epoxy composite with optimal thermal properties. RSC Adv 5:20376-20385. https://doi.org/10.1039/c4ra15881d

[29] Wang R, Zhuo D, Weng $Z$ et al (2015) A novel nanosilica/graphene oxide hybrid and its flame retarding epoxy resin with simultaneously improved mechanical, thermal conductivity, and dielectric properties. J Mater Chem A 3:9826-9836. https://doi.org/10.1039/c5ta00722d

[30] Mancinelli P, Heid TF, Fabiani D et al (2013) Thermal in situ reduction of graphene oxide in epoxy-based nanodielectrics: influence on dielectric properties. In: Annual report-conference on electrical insulation and dielectric phenomena, CEIDP, pp 768-771

[31] Mancinelli P, Fabiani D, Saccani A et al (2013) Preparation and dielectric behavior of epoxy resin containing graphene oxide. In: Proceedings of IEEE international conference on solid dielectrics, ICSD, pp 915-918

[32] Mancinelli P, Heid TF, Fabiani D et al (2013) Electrical conductivity of graphene-based epoxy nanodielectrics. In: Annual report-conference electrical insulation dielectric phenomena, CEIDP, pp 772-775. https://doi.org/10.1109/ce idp.2013.6748282

[33] Paredes JI, Villar-Rodil S, Martínez-Alonso A, Tascón JMD (2008) Graphene oxide dispersions in organic solvents. Langmuir 24:10560-10564. https://doi.org/10.1021/la 801744a

[34] Vryonis O, Andritsch T, Vaughan AS, Lewin PL (2017) On the effect of solvent method processing on epoxy resin systems: a molecular dynamics study. In: 2017 IEEE conference on electrical insulation and dielectric phenomenon (CEIDP). IEEE, pp 509-512

[35] Menczel JD, Bair HE, Reading M et al (2009) Differential scanning calorimetry (DSC). In: Menczel JD, Prime RB (eds) Thermal analysis of polymers: fundamentals and applications. Wiley, New York, pp 7-239

[36] Virtanen S, Vaughan AS, Yang S et al (2017) Electrical conductivity and moisture uptake studies of low density polyethylene octylnanosilica composite. In: Proceedings of the Nordic insulation symposium. https://doi.org/10.5324/n ordis.v0i25.2368

[37] Geyer RG (1990) Suitable dielectric reference materials. In: Lyons JW (ed) Dielectric characterization and reference materials. Technical Note-1338. NIST Publications, Gaithersburg, MD, pp 85-94

[38] Sharmila TKB, Nair AB, Abraham BT et al (2014) Microwave exfoliated reduced graphene oxide epoxy nanocomposites for high performance applications. Polym (Guildf) 55:3614-3627. https://doi.org/10.1016/j.polymer.2014.05. 032

[39] Esmaeili A, Entezari MH (2014) Facile and fast synthesis of graphene oxide nanosheets via bath ultrasonic irradiation. J Colloid Interface Sci 432:19-25. https://doi.org/10.1016/j. jcis.2014.06.055

[40] Vassilikou-Dova A, Kalogeras IM (2008) Dielectric analysis (DEA). Thermal analysis of polymers: fundamentals and applications. Wiley, New York, pp 497-613

[41] Nakka JS, Jansen KMB, Ernst LJ (2013) Tailoring the viscoelasticity of epoxy thermosets. J Appl Polym Sci 128:3794-3806. https://doi.org/10.1002/app.38435

[42] Gillham JK (1986) Formation and properties of thermosetting and high $\mathrm{Tg}$ polymeric materials. Polym Eng Sci 26:1429-1433. https://doi.org/10.1002/pen.760262012

[43] González-González M, Cabanelas JC, Baselga J (2012) Applications of FTIR on epoxy resins-identification, monitoring the curing process, phase separation and water 
uptake. In: Theophanides TM (ed) Infrared spectroscopymaterials science, engineering and technology. InTech, London, pp 261-284

[44] Nikolic G, Zlatkovic S, Cakic M et al (2010) Fast fourier transform IR characterization of epoxy GY systems crosslinked with aliphatic and cycloaliphatic EH polyamine adducts. Sensors 10:684-696. https://doi.org/10.3390/ s100100684

[45] Chike KE, Myrick ML, Lyon RE, Angel SM (1993) Raman and near-infrared studies of an epoxy resin. Appl Spectrosc 47:1631-1635. https://doi.org/10.1366/0003702934334714

[46] Fothergill JC (2010) Electrical Properties. Dielectric polymer nanocomposites. Springer, Troy, NY, USA, pp 197-228

[47] Vryonis O, Anastassopoulos DL, Vradis AA, Psarras GC (2016) Dielectric response and molecular dynamics in epoxy-BaSrTiO3 nanocomposites: effect of nanofiller loading. Polym (Guildf) 95:82-90. https://doi.org/10.1016/j.pol ymer.2016.04.050

[48] Singha S, Thomas M (2008) Dielectric properties of epoxy nanocomposites. IEEE Trans Dielectr Electr Insul 15:12-23. https://doi.org/10.1109/T-DEI.2008.4446732

[49] Gonon P, Boudefel A (2006) Electrical properties of epoxy/ silver nanocomposites. J Appl Phys 99:2. https://doi.org/10. $1063 / 1.2163978$
[50] Psarras GC (2010) Conductivity and dielectric characterization of polymer nanocomposites. Polymer nanocomposites: physical properties and applications. Woodhead Publishing Limited, Cambridge, pp 31-69

[51] Kremer F, Schönhals A (2003) Molecular and Collective Dynamics of (Polymeric) Liquid Crystals. Broadband dielectric spectroscopy. Springer, Berlin, pp 385-432

[52] Schönhals A (2003) Molecular dynamics in polymer model systems. Broadband dielectric spectroscopy. Springer, Berlin, pp 225-293

[53] Schönhals A, Kremer F (2003) Theory of Dielectric Relaxation. Broadband dielectric spectroscopy. Springer, Berlin, pp 1-33

[54] Hsu CH, Hsu MH, Chang KC et al (2014) Physical study of room-temperature-cured epoxy/thermally reduced graphene oxides with various contents of oxygen-containing groups. Polym Int 63:1765-1770. https://doi.org/10.1002/pi.4763

[55] Rafiee MA, Rafiee J, Wang Z et al (2009) Enhanced mechanical properties of nanocomposites at low graphene content. ACS Nano 3:3884-3890. https://doi.org/10.1021/ nn9010472 